\title{
The Phase-Transition Beneath the Solar Surface
}

\author{
Frederick J Mayer \\ Mayer Applied Research Inc., 1417 Dicken Drive, Ann Arbor, MI, USA
}

\section{Article Info}

\author{
*Corresponding author: \\ Frederick J Mayer \\ President \\ Mayer Applied Research, Inc. \\ 1417 Dicken Drive \\ Ann Arbor, M \\ USA \\ Tel: + 7346623841 \\ E-mail: fred@frederickjmayer.net
}

Received: March 12, 2021

Accepted: April 02, 2021

Published: April 09, 2021

Citation: Mayer FJ. The Phase-Transition Beneath the Solar Surface. Int J Cosmol Astron Astrophys. 2021; 3(1): 121-124. doi: 10.18689/ijcaa-1000125

Copyright: (c) 2021 The Author(s). This work is licensed under a Creative Commons Attribution 4.0 International License, which permits unrestricted use, distribution, and reproduction in any medium, provided the original work is properly cited.

Published by Madridge Publishers

\begin{abstract}
This paper discusses the implications of the recently-identified composite particle (the tresino and its formation energy) on the unexplained heating and eruptions in the solar corona.
\end{abstract}

Keywords: Tresinos; Solar corona; Corona disturbances.

\section{History \& Background}

As early as the 1990s, during discussions about energy production in the early universe, my late colleague John Reitz and I had uncovered the possibility of a new particle (i.e., a previously overlooked composite particle) in the early years of the development of atomic and nuclear physics. We felt that this oversight would have manifested itself in observations in various physical problems regarding energy generation. The formation of this composite particle, which we named the tresino, would result in the release of a significant amount of "binding" energy. Thereafter our research focussed on the implications of the energy released from the earth [1] and the implications relating to the energy in the Early Universe [2]. This paper examines the implications of tresino energy generation in the sun.

\section{Solar Paradoxes}

The most well-known solar paradox is how the corona can be so hot $(\approx 100 \mathrm{eV})$ while the surface is so relatively cool $(\approx 0.5 \mathrm{eV})$. Of course, there is an enormous amount of energy being produced by nuclear reactions in the solar core, but it has never been clear how these reactions could produce the high-temperatures observed in the corona. Years of magnetic field observations in the corona prompted many researchers to look to the magnetic fields as the energy source but this has generally turned out to be too difficult to understand and largely inconclusive. So, if there is some other source of energy powering the corona heating and disturbances, what might it be? This paper proposes that the phase-transition of the recently-identified composite particle (the tresino) may be that source.

\section{Brief Comments about Tresinos and This Paper}

As I mentioned above, my late colleague John Reitz and I began to realize many years ago that there was an overlooked set of Compton-scale composite particles, the most important of which is one that we named the tresino.

The illustration below shows the tresino transition as it forms out of the equilibrium hydrogen plasma (depicted on the left-hand side). In the illustration, protons are blue, electrons are green, and the tresino is shown with a red-circle around the newly-formed composite. 


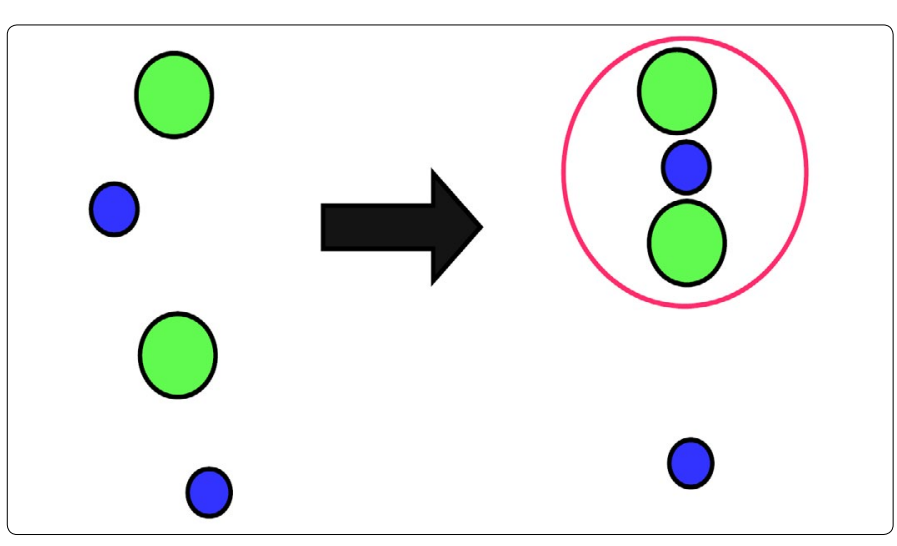

This picture shows the baryon density (here protons) changing through the transition from its plasma phase to the new phase where the protons and electrons change into nearly equal numbers of protons and tresinos. (Note: The illustration is similar in character to Figure. 4 of [3].

The reader will find several of our published papers on the tresino in the references, but briefly, the tresino is composed of two electrons bound to one proton; it was recognized as a "bound state" so, at formation, it would release its binding energy and would remain in this configuration unless or until the binding energy was in some way re-introduced. We had especially wanted to examine tresinos and the role they might play in the Early Universe [3]. This work later led to additional connections in other physics research arenas. In this early paper we developed a Baryon Phase Transition cosmology [6] that was so-named because of the tresino phase-transition. Furthermore, we needed to understand the equilibrium statistical-mechanics (Saha-Boltzmann Equilibrium Combination) for tresino composites [3] which, as will be seen below, is important in this paper as well. Note that the tresinos are composed of well-understood particles, electrons and protons, but in a new combination held together by well-understood forces that has broad implications.

Readers unfamiliar with Compton-composites or our theory of tresinos may find it useful to peruse my reference [5] about hidden baryons; and perhaps some of the references therein. Tresinos are held together in a balance between electrostatic attraction of two electrons to one proton at larger distances, and dipole-dipole magnetic repulsion, at shorter distances. Because the electrons are strongly correlated, the tresino composite is a resonant structure.

The origin of the present paper was initiated by a couple of questions that arose during a physics seminar soon after our first paper on the tresino phase-transition was published. One physicist asked "isn't this a new state of matter?" A second asked "wouldn't the sun be a good place to search for tresinos?" As it turned out, the answer to both of their questions is "yes". In fact, their questions inspired the present work even though I did not have the answers to their questions at the time of the seminar.

In [3] we had recognized and even mentioned that the plasma parameters at the phase-transition in the Early Universe were remarkably close to those of the solar transition-region; this closeness did suggest a possible connection between these phenomena. But examining the complexities of the solar corona didn't appear to be a fruitful direction to proceed at the time.
It's important for the reader to understand that, while the tresino phase-transition and the solar transition region, which have similar names and are connected, are distinct and separate. Finally, it should be emphasized that the formation of one tresino releases $3.7 \mathrm{keV}$ of energy, a large amount on the scale of, say, hydrogen recombination. Therefore, a large number of tresino formations will yield large amounts of energy; this is a crucial observation of this paper.

\section{Solar data expanded}

After considerable searching, I found the excellent, highly-detailed paper by [4] that provided the data that were required to search for the tresino phase-transition in the sun - specifically the plasma parameters.

I thank Dr. Eugene Avrett (Harvard-Smithsonian Center for Astrophysics) and his colleague, Professor Petr Heinzel (Czech Academy of Sciences), for helping me construct a Mathematica-based computer file from the large tables of the Atmospheric Parameters for Model C7. This data file starts just above the corona through the solar transition-region to depths many kms below the surface. Figure. 1 is a plot of the data region examined in this work. Note that the index $n$ refers to the row number in the $\mathrm{C} 7$ atmospheric data file and $\mathrm{km}$ is the depth beneath the solar transition zone at $\mathrm{n}=130$, "generally considered to be the surface of the sun".

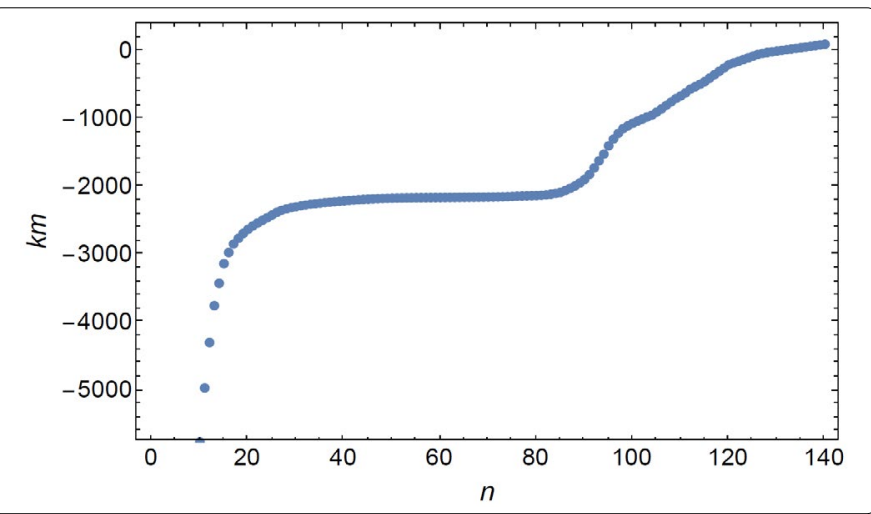

Figure. 1. Region of data showing the depth $k m$ vs. $n$ of the $C 7$ model.

\section{Locating the Tresino Phase-Transition}

The density and temperature data from the $\mathrm{C} 7$ model can be introduced into the Saha-Boltzmann equilibrium formula just as we did in the Early Universe calculation in [3].

$$
\frac{x(2 x-1)^{4}}{1-x}=\frac{7.4 \times 10^{99} e^{-3700 / T_{\mathrm{ev}}} T_{\mathrm{ev}}^{6}}{n_{o}^{4}}
$$

where $n_{e} / n_{o}=x$ is the ratio of electrons to hydrogen, $n_{p} / n_{0}=(2 x-1)$ is the ratio of protons to hydrogen, and $n_{t} / n_{o}=(1-\mathrm{x})$ is the ratio of tresinos to hydrogen.

It is straightforward to choose values of $n$ to obtain the plasma parameters for substituting in Equation (1) to see where the tresino phase-transition appears. The result of the search is presented in Figure. 3. Notice that the tresino phasetransition takes place at $\mathrm{n} \approx 27$. From Figure 1 or directly from the $\mathrm{C} 7$ data table it is found that this corresponds to a depth of about $2350 \mathrm{~km}$ and the plasma parameters at this location have $T_{e v} \approx 17$ and hydrogen density $n_{0} \approx 4700 / \mathrm{cc}$. 


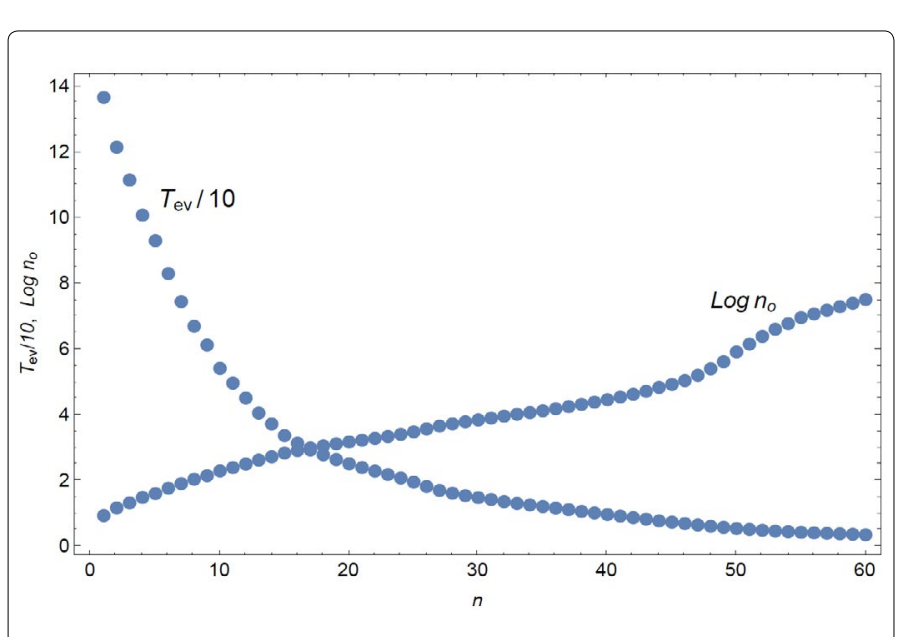

Figure 2. The combined plot of the temperature in ev and the hydrogen plasma density $n_{o}$ over the region examined for locating the phasetransition.

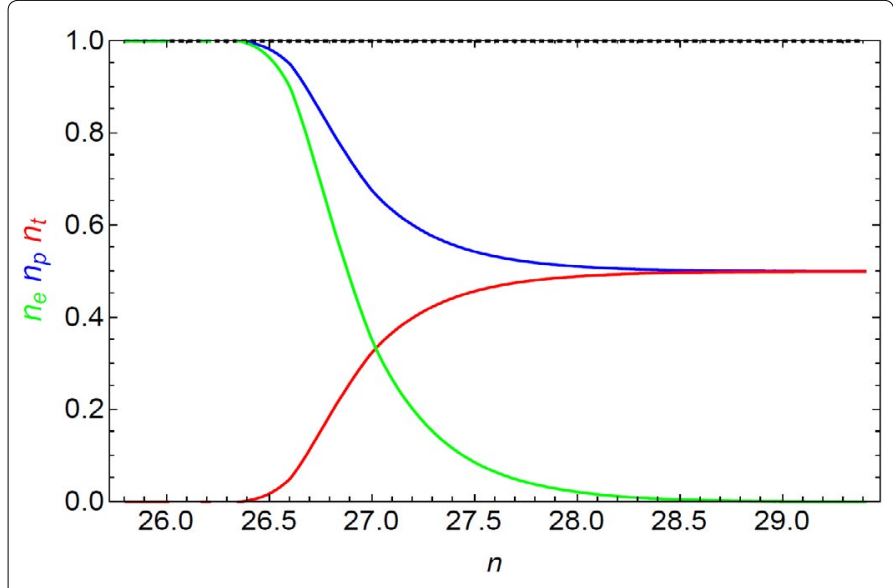

Figure. 3. A plot presenting color-coded (normalized) densities of electrons, protons and tresinos located at the phase-transition.

Figure. 3 shows that the phase-transition conserves the particle species as does the illustration presented above. This solar tresino phase-transition result may be readily compared to that of Figure. 4 of [3]; although these two transitions are on very different time and space scales. It is quite interesting that during the tresino phase-transition in the Early Universe, the plasma density and temperature were indexed by $z_{1}$ the red-shift parameter, (essentially meaning how far away in time that it occurred). In the sun, however, it is indexed by $n$, the distance from the solar transition region (essentially how far away it was in space).

\section{Tresino Phase-Transition Energies}

The tresino phase-transition just presented was found to take place quite far under the solar surface. How this happens has to be discussed and understood. To me, the picture that seems most probable is that a large hydrogen plasma "bubble" is released from the sun's deep interior and drifts toward the surface. It finally arrives at the location of the tresino phase-transition and then releases the energy of the many transitions from hydrogen plasma into tresinos. The scale of this energy release can be enormous. The size will have to be on the order of many tens or hundreds of kms in radius. Recalling that each transition releases $3.7 \mathrm{keV}$, the total energy release would have to be on the order of $\left(10^{5}\right)^{3} \times$ $3.7 \times 10^{3} \times n_{t}$ ev per $\mathrm{km}^{3}$ where $n_{t}$ is the number of tresinos generated. For the example presented in the sections above, the energy generated is somewhere between 70 to 95 kilojoules $/ \mathrm{km}^{3}$, assuming all of the hydrogen encountered was converted to tresinos. However, it seems quite likely that the hydrogen "bubble" would be many kms in transverse directions so the total energies would be many times, perhaps thousands to millions of times, larger. Recall that the energy released is found in streams of protons and tresinos as seen in Figure. 3. The current streams will carry their associated magnetic fields as well as any entrained hydrogen plasma during its explosive expansion. In addition, the streams will be mostly unaffected by the overlying atmosphere of the sun due to its extremely low density. Hence, the streams and entrained plasma will reach into the solar corona and beyond producing solar flares and other disturbances we observe on the surface of the sun. As I had noted in [7], the kinetic energies of these charged-streams were either at or above the escape velocity of the sun. Furthermore the streams of currents would interact with each other as well as the ambient solar magnetic field as they drag some amount of background plasma along with them. Because of the large currents and magnetic energy involved, they give rise to the magnetohydrodynamic eruptions and chaos so often observed in the corona and above.

Finally, there will be a range of energies produced depending upon the sizes of the hydrogen "bubbles" in transverse directions. In the case of a smaller but continuous flow of "bubbles", a background heating of the corona would be expected; whereas, in the case of much larger "bubbles", the explosion of the outer solar material would produce the events generally referred to as coronal mass ejections.

In other words, the process of tresino formation and its energy generation, even though arising well below the solar surface, may explain the mysterious characteristics and behavior observed in the sun's corona and beyond.

\section{Acknowledgments}

I dedicate this paper to my mentor, collaborator, and friend Dr. John R. Reitz, without whose efforts this work would not have become possible.

\section{Addendum: The Phase Transition at the Solar Surface}

The tresino transition of the earlier pages was found deep under the solar surface in a region that had well resolved plasma quantities but it was not the only location wherein the transition is found. In particular, at the apply-named "solar transition region" it appears that the same phase-transition obtains there as well. But here the transition is much more abrupt (more like a "step-function") and is less resolved in the Avrett \& Loeser data file. This addendum is provided in order to make clear how this occurs. 


\section{A 1. The Generalized Tresino Transition}

Recall Eq.(1) that the tresino-transition takes the form:

$$
\frac{x(2 x-1)^{4}}{1-x}=\frac{7.4 \times 10^{99} e^{-3700 / T_{\mathrm{ev}}} T_{\mathrm{ev}}^{6}}{n_{o}^{4}}
$$

Let us consider the general case of Eq.(2). First, the lefthand side is required, but in order to simplify the solution, I average the LHS over the domain over which $x$ varies, namely from 0 to 1 (actually 0 to 0.999 to avoid the singularity at $x=1$ ). That average results in the LHS average value of 4.0541 for the general transition case. Then, solving Eq. (2) for no by taking the LHS equal to its average value results in:

$$
n_{o}=6.5363 \times 10^{24} \exp \left(-925 / T_{\mathrm{ev}}\right) T_{\mathrm{ev}}{ }^{3 / 2}
$$

or in degrees Kelvin (K)

$$
n_{o}=5.231 \times 10^{18} \exp \left(-1.073 \times 10^{7} / K\right) K^{3 / 2}
$$

Eq. (4) is a relationship between the hydrogen density no and the temperature $(K)$ for the generalized tresinotransition that was sought.

\section{A 2. Comparison with Model C7 Data}

It is straightforward to compare the data in the Avrett \& Loeser Model C7 at the solar surface (height $\approx 2100 \mathrm{~km}$ ) as presented in their Figure 8. For heights below this point, the hydrogen density no is quite high and the temperature is quite low: this is not consistent with transition evaluation given by Eq.(4). But above this height, the temperature is quite high and the hydrogen density no is quite low. This can be noted in the plot below.

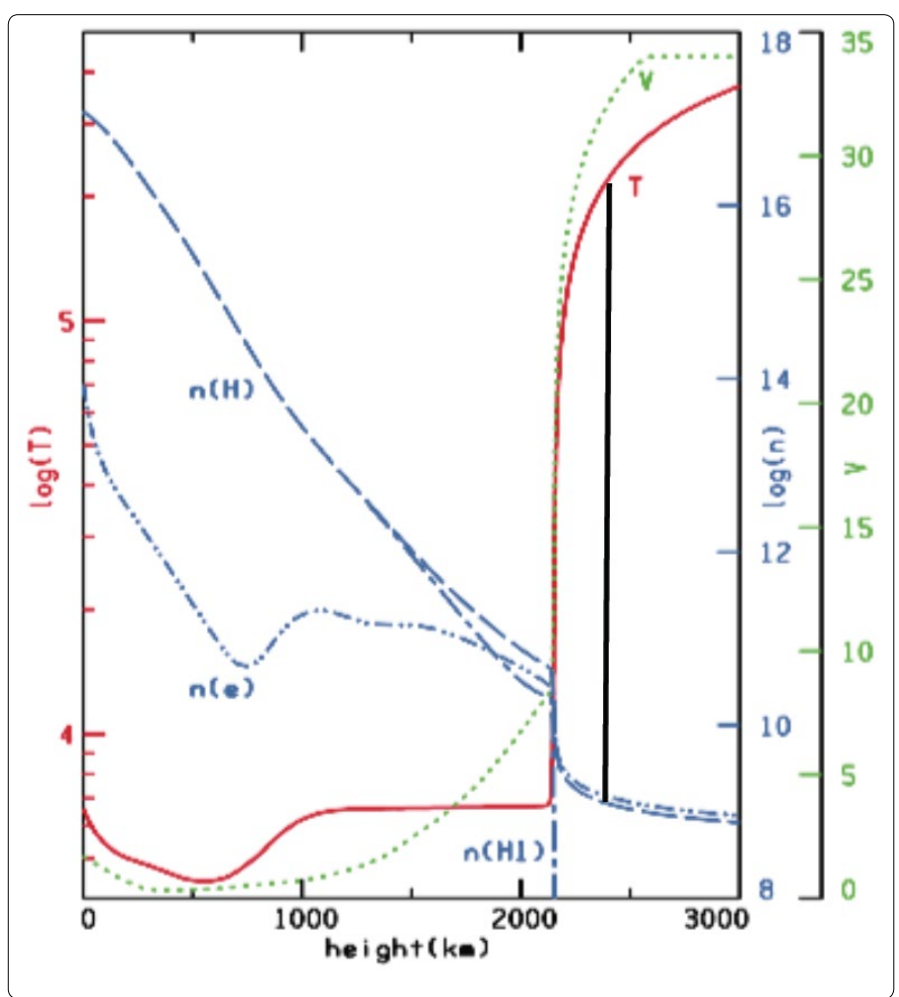

Figure 4. A reproduction of Figure 8 of Avrett \& Loeser Model C7 where the (added) black-line represents a solution to the tresino-transition. Note, the transition is first completed at a point closer to the solar surface than indicated by the black-line.
The higher temperatures are a result of the transition delivering the extra energy to the outflow by converting the normal hydrogen plasma to tresino and proton streams while conserving the total numbers of electrons and protons in accord with the schematic presented at the beginning of this paper.

In summary, the tresino-phase transition has been shown to operate both deep under the solar surface as well as at the surface creating the "solar corona" producing the well-observed solar phenomena.

\section{References}

1. Mayer FJ. Geophysical Implications of Tresino Formation: A Narrated Review. Int J Earth Sci Geol. 2020a; 2(1): 86-89. doi: 10.18689/ijeg1000110

2. Mayer FJ. Cosmic Implications of Tresino Formation: A Narrated Review. Int J Cosmol Astron Astrophys. 2020b; 2(1): 112-114. doi: 10.18689/ijcaa-1000123.

3. Mayer FJ, Reitz JR. Compton Composites Late in the Early Universe. Galaxies. 2014; 2(3) 382-409. doi: 10.3390/galaxies2030382

4. Avrett EH, Loeser R. Models of the Solar Chromosphere and Transition Region from SUMER and HRTS Observations: Formation of the Extreme-Ultraviolat Spectrum of Hydrogen, Carbon, and Oxygen; ApJS, 2008; 175, 229.

5. Frederick Mayer. Hidden baryons: The physics of Compton composites. EPL. 2016; 114(6): 69001-69007. doi: 10.1209/02955075/114/69001

6. Mayer F. The Baryon Phase-Transition Model and the too strange Standard Model of Cosmology. universe. 2017; 3(1): 318- 324. doi: 10.3390/universe 3010018

7. Mayer F. Dark rotors in the late universe. Heliyon. 2015; e00039. doi: 10.1016/j.heliyon.2015.e00039 